\title{
Asm8, a specific LAL-type activator of 3-amino-5-hydroxy- benzoate biosynthesis in ansamitocin production
}

\author{
PAN WenQin, KANG QianJin, WANG Lei, BAI LinQuan \& DENG ZiXin \\ State Key Laboratory of Microbial Metabolism and School of Life Sciences \& Biotechnology, Shanghai Jiao Tong University, \\ Shanghai 200240, China
}

Received April 27, 2013; accepted May 28, 2013

\begin{abstract}
The highly potent antitumor agent ansamitocin P3 is a macrolactam isolated from Actinosynnema pretiosum ATCC 31565. A 120-kb DNA fragment was previously identified as the ansamitocin biosynthetic gene cluster, and contains genes for polyketide assembly, precursor synthesis, post-polyketide synthesis modification, and regulation. Within the biosynthetic gene cluster, asm8 encodes an 1117-amino-acid protein with a high degree of similarity to the large ATP-binding LuxR family-type regulators. In the current study, we determined that inactivation of asm8 by gene replacement in ATCC 31565 resulted in the complete loss of ansamitocin production, and that complementation with a cloned asm 8 gene restored ansamitocin biosynthesis. Interestingly, the disruption of asm 8 decreased the transcription of genes responsible for 3-amino-5-hydroxybenzoate (AHBA) formation, the starter unit required for ansamitocin biosynthesis. Subsequently, feeding of exogenous AHBA to the asm 8 mutant restored ansamitocin biosynthesis, which showed that Asm8 is a specific positive regulator in AHBA biosynthesis. In addition, investigation of asm8 homologs identified two new ansamitocin producers, and inactivation of the $a s m 8$ homolog in $A$. pretiosum ATCC 31280 abolished ansamitocin production in this strain. Characterization of the positive regulator Asm8 and discovery of the two new ansamitocin producers paves the way for further improving production of this important antitumor agent.
\end{abstract}

ansamitocins, regulation, AHBA, Actinosynnema pretiosum, LuxR family

Citation: $\quad$ Pan W Q, Kang Q J, Wang L, et al. Asm8, a specific LAL-type activator of 3-amino-5-hydroxy-benzoate biosynthesis in ansamitocin production. Sci China Life Sci, 2013, 56: 601-608, doi: 10.1007/s11427-013-4502-4

Like their plant-derived maytansinoid counterparts [1], ansamitocins (e.g., ansamitocin P-3; AP-3) demonstrate potent antitumor activity by specifically inhibiting eukaryotic mitosis [2]. The antibody-conjugated drug Kadcyla uses maytansinoid as the active compound, and was approved in February 2013 by the United States Food and Drug Administration for treating breast cancer [3]. Produced by Actinosynnema pretiosum subsp. auranticum ATCC 31565 (hereafter referred to as ATCC 31565), these microbial metabolites belong to an important class of polyketide natural products called ansamycins [2]. All ansamycin antibiotics

*Corresponding author (email: bailq@ @jtu.edu.cn) are macrolactams, and are characterized by an amide-linkageterminated benzoic acid or a naphthalenic chromophore bridged by an aliphatic polyketide. Typical ansamycins include rifamycin, ansamitocin, and macbecins.

Biosynthesis of ansamycin antibiotics involves the formation of 3-amino-5-hydroxybenzoate (AHBA) as the starter unit for the assembly of the polyketide chain. The first three steps of the AHBA pathway are very similar to the shikimate pathway, but later steps are differentiated by the introduction of glutamine-derived nitrogen, to give 3,4-dideoxy-4-amino-D-arabino-heptulosonic acid 7-phosphate (aminoDAHP). Cyclization and dehydration of aminoDAHP generates 5-deoxy-5-amino-3-dehydroquinic acid 
(aminoDHS), which is finally aromatized by the enzyme AHBA synthase to give AHBA [4,5]. Sequence analysis of the ansamitocin biosynthetic gene cluster identified 52 open reading frames in two separate regions of 83 and $12 \mathrm{~kb}$ [6]. Among these genes, asm47, -23, -44, -45, and -22 are homologs of rifG, $-J,-L,-M$, and $-N$, respectively, from the rifamycin biosynthetic gene cluster [6]. Both asm24 and asm43 are homologs of rifK [5] (Figure 1A). Moreover, genes asm43-47 form an operon, and asm23 and 24 are transcribed from a divergent promoter region (Figure 1B).

The biosynthetic mechanism of ansamitocins has been studied extensively [6-9]. However, little is known about the regulation of ansamitocin biosynthesis. Previous sequencing of the ansamitocin biosynthetic gene cluster has revealed the presence of a set of regulatory genes. Three genes, asm2, -29, and -34 , encode TetR family proteins, while asm31, -39, and -40 encode a sigma factor, sigma factor antagonist, and anti-sigma antagonist, respectively. The protein encoded by asm 18 is homologous to a SARP (Streptomyces antibiotic regulatory protein) family protein. The remaining two proteins, encoded by asm 8 and asm 48 , are similar to LuxR or LAL family (large ATP-binding regulator of the LuxR family) regulatory proteins.

A previous study showed that in-frame deletion of asm2 caused an over-production of ansamitocins [10]. More interestingly, AP-3 production was also increased by constitutive over-expression of asm2 and asm39 [11].

Here, the function of asm 8 was studied through gene in- activation, complementation, transcriptional analysis, and feeding experiments. Results showed that $a s m 8$ is a positive regulator of ansamitocin production, specifically the formation of the AHBA starter unit, in ATCC 31565. Further investigation of asm8 homologs through gene comparison and gene inactivation identified two additional novel ansamitocin producers.

\section{Materials and methods}

\subsection{Bacterial strains, cloning vectors, and cultivation}

Strains and plasmids used in this study are listed in Table S1. Actinosynnema strains were cultivated at $30^{\circ} \mathrm{C}$ and 220 $\mathrm{r} \mathrm{min}^{-1}$ in liquid tryptic soy broth medium (supplemented with $1 \%$ yeast extract and $10.3 \%$ sucrose) for $2 \mathrm{~d}$. Fermentation was carried out on yeast and malt extract with glucose (YMG) agar plates at $30^{\circ} \mathrm{C}$ for $7 \mathrm{~d}$. The same medium was supplemented with apramycin when used for growth and/or metabolite production of the asm8 mutant, PW201. E. coli strains DH10B (Invitrogen, New York) and ET12567 (pUZ8002) were used as hosts for plasmid construction and E. coli-Streptomyces conjugation [12], respectively. pJTU1278 was used for gene inactivation [13], and pBluescript KS(-) (Agilent Technologies Inc., Santa Clara, CA, USA) and pUC18 (Thermo Fisher Scientific Inc., Waltham, MA, USA) were used for gene cloning and DNA sequencing. pRSET-B (Invitrogen, New York) was used for protein over-expression
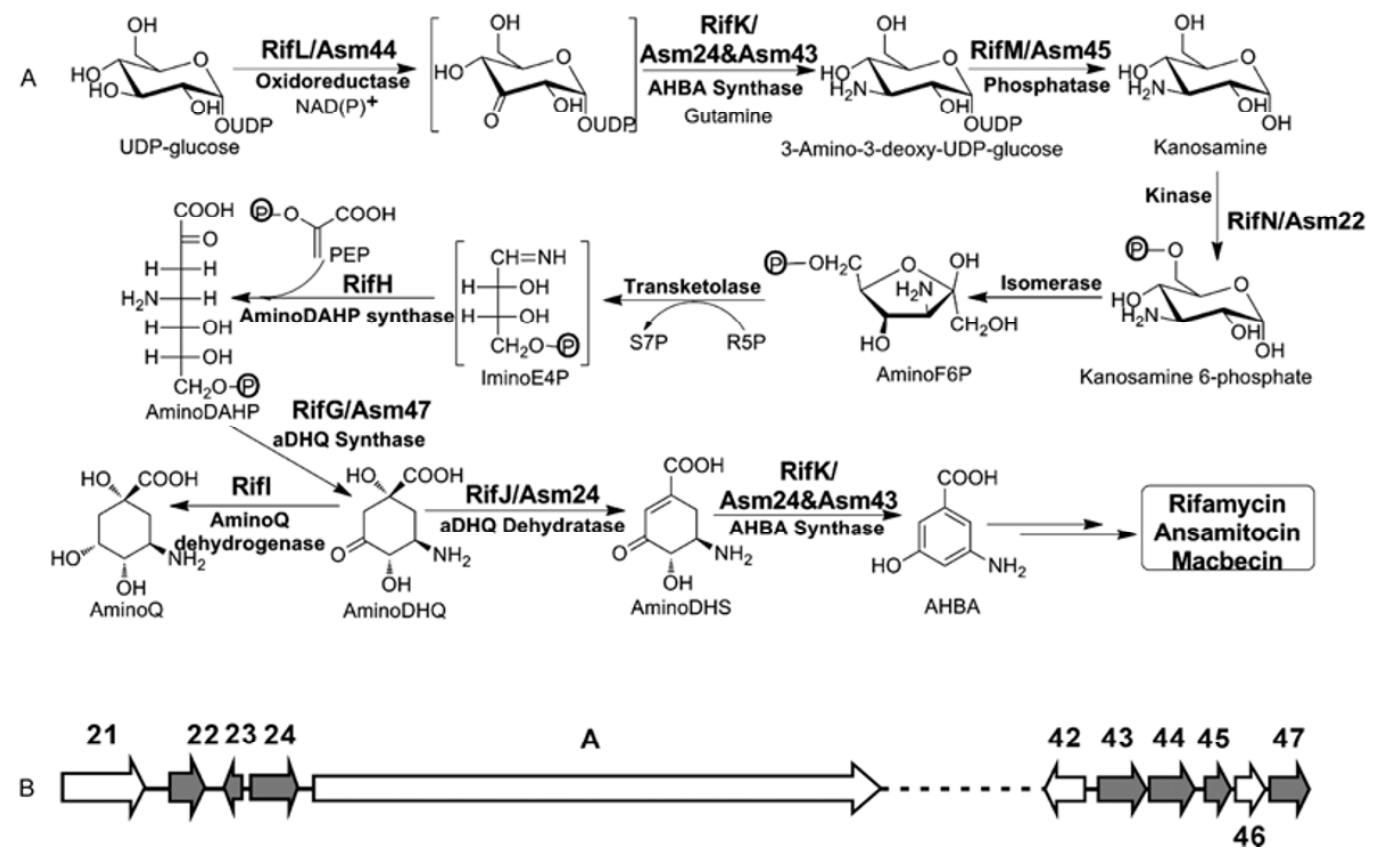

Figure 1 Proposed biosynthetic pathway of AHBA, and organization of the AHBA biosynthetic gene cluster. A, Proposed pathway for AHBA biosynthesis. Gln (glutamine), aminoF6P (3-amino-3-deoxy-D-fructose 6-phosphate), iminoE4P (1-deoxy-1-imino-D-erythrose 4-phosphate), PEP (phosphoenolpyruvate), aminoDAHP (3,4-dideoxy-4-amino-D-arabino-heptulosonic acid 7-phosphate), aminoDHQ (5-deoxy-5-amino-3-dehydroquinic acid), aminoDHS (5-deoxy-5-amino-3-dehydroshikimic acid), AHBA (3-amino-5-hydroxybenzoate). B, Organization of AHBA biosynthetic gene cluster (asm21-24 and asm42-47) in ATCC 31565. 
in E. coli. Shuttle vectors pIB139 [14] and pSET152 [15] were used for gene complementation.

\subsection{Genetic manipulation}

Standard genetic techniques using E. coli and in vitro DNA manipulation were performed as described by Sambrook et al. [16]. Recombinant DNA techniques in Actinosynnema species and isolation of Actinosynnema DNA were performed as described previously [17].

\subsection{Disruption of $a s m 8$ using Redirect Technology}

A 7.0-kb Sac I/BamH I fragment from cosmid 3C11 [6] was cloned into pJTU1278, to generate pJTU851. The 3.40-kb asm8 gene within pJTU851 was replaced with an aac3(IV)-oriT cassette, to generate pJTU852, using Redirect Technology according to a previously published method [18]. The $\operatorname{aac}(3) I V$-oriT cassette was amplified from pIJ773 using primer pair asm8-tgt-F (5'-ATGCGGCTGGTGGACCGCGACGGGCAGTGCGAGGCGCTGattccggggatccgtcgacc-3') and asm8-tgt-R (5'-TCAGGCGGGGGTGGTCAGGTCGGCGTGCAGGGTGGTGGGtgtaggctggagctgcttc$3^{\prime}$ ) (pIJ773 homologous sequences are in lowercase). For a marker-free gene replacement, pJTU852 was digested with $X b a$ I and then re-ligated to generate pJTU886, in which the aac(3)IV-oriT cassette was replaced with an 81-bp "scar" sequence. pJTU886 was used in conjugation with an apramycin-resistant asm 8 mutant to generate the marker-free mutant PW1, which was verified by PCR amplification with the primers asm8-Det-F (5'-AGTGGGGGTGCGGTGGT$\left.3^{\prime}\right)$ and asm8-Det-R (5'-CTGGTCGGGTCGGGGTC-3').

\subsection{Trans-complementation of the mutant PW1 with cloned asm8}

For the complementation of the PW1 $\Delta a s m 8$ mutant, a 3.80-kb Nru I fragment from pJTU851, containing asm8 and its promoter region, was cloned into pSET152. The resulting plasmid, pJTU3101, was then transferred by conjugation from E. coli ET12567 (pUZ8002) to PW1, as described previously [19].

\subsection{Fermentation, purification, and analysis of ansa- mitocins}

Seven-day YMG agar plate cultures of the wild-type ATCC 31565 strain and the PW1 mutant were extracted with ethyl acetate. The extract was concentrated under reduced pressure, and was further extracted with $1 \mathrm{~mL}$ methanol. The methanol extract was analyzed by liquid chromatography-mass spectrometry (LC-MS) using an Agilent 1100 series LC/MSD Trap system [8]. High performance liquid chromatography (HPLC) was operated at a flow rate of 0.45 $\mathrm{mL} \min ^{-1}$ with methanol-water $(70 / 30, \mathrm{v} / \mathrm{v})$ on a Calesil
ODS-100 (4.6 mm×150 mm, $5 \mu \mathrm{m})$ column. An ion-trap mass spectrometer was operated with the electrospray ionization source in positive ion mode. The drying gas flow was $6 \mathrm{~L} \mathrm{~min}^{-1}$, and the nebulizer pressure was $30 \mathrm{psi}$. The drying gas temperature was $325^{\circ} \mathrm{C}$.

\subsection{RNA isolation and quantitative reverse transcrip- tion-polymerase chain reaction (qRT-PCR) analysis}

A two-day culture of A. pretiosum mycelia was collected from YMG agar plates and disrupted using glass beads (150-212 $\mu \mathrm{m}$, Sigma) in a Precellys homogenizer (6500 $\mathrm{r} \min ^{-1}, 2 \times 20 \mathrm{~s}$; Peqlab). RNA was isolated using a Total RNA Isolation Kit (SBS Genetech, Shanghai) and then treated with RNase-free DNase I (Thermo Fisher Scientific Inc.) to eliminate possible chromosomal DNA contamination.

Equal amounts of purified RNA (3 $\mu \mathrm{g})$ were used to generate cDNA using RevertAid $\mathrm{H}$ Minus reverse transcriptase and random hexamer primers, as described by the manufacturer (Thermo Fisher Scientific Inc.). The conditions were as follows: first strand cDNA synthesis, $42^{\circ} \mathrm{C}$ for $1 \mathrm{~h}$, followed by $70^{\circ} \mathrm{C}$ for $10 \mathrm{~min}$. One microliter of synthesized cDNA was then used as template for each qRT-PCR, which was performed following the Maxima SYBR Green/ROX qPCR master mix protocol (Thermo Fisher Scientific Inc.). The conditions were as follows: $50^{\circ} \mathrm{C}$ for $20 \mathrm{~s}$, $95^{\circ} \mathrm{C}$ for $10 \mathrm{~min}$, followed by 40 cycles of $95^{\circ} \mathrm{C}$ for $15 \mathrm{~s}$ and $60^{\circ} \mathrm{C}$ for $1 \mathrm{~min}$. The DNA gyrase gene gyrB was used as an internal control. The transcription of 45 genes in the biosynthetic gene cluster was analyzed.

\section{Results}

\subsection{Asm8 is positively involved in ansamitocin biosyn- thesis}

To investigate the function of asm8, an in-frame deletion of asm 8 was performed in E. coli using Redirect Technology [18], followed by two rounds of conjugation with ATCC 31565 (Figure 2A). Three thiostrepton-sensitive apramycinsensitive (Thio ${ }^{\mathrm{S}} \mathrm{Apr}^{\mathrm{S}}$ ) mutants (designated PW1-1-PW1-3) were obtained. Total genomic DNA was extracted from the mutants and the wild-type was compared by PCR. The wild-type gave a 3.80-kb amplified product, whereas amplicons from the mutants were only $0.60 \mathrm{~kb}$ (Figure 2B), confirming that a 3354-bp internal DNA fragment, corresponding to asm8, had been replaced by an 81-bp scar fragment in the mutant genomes. ATCC 31565 and the PW1 mutants were incubated in fermentation medium for $7 \mathrm{~d}$. The fermentation broths were then analyzed by LC-MS. AP-3, with a retention time of $19.7 \mathrm{~min}$ and an $\mathrm{m} / \mathrm{z}$ of 635 , was not detected in the fermentation broths of the PW1 mutants (Figure 2C).

To verify that the loss of AP-3 was caused only by the asm8 inactivation, mutant PW1 was trans-complemented 

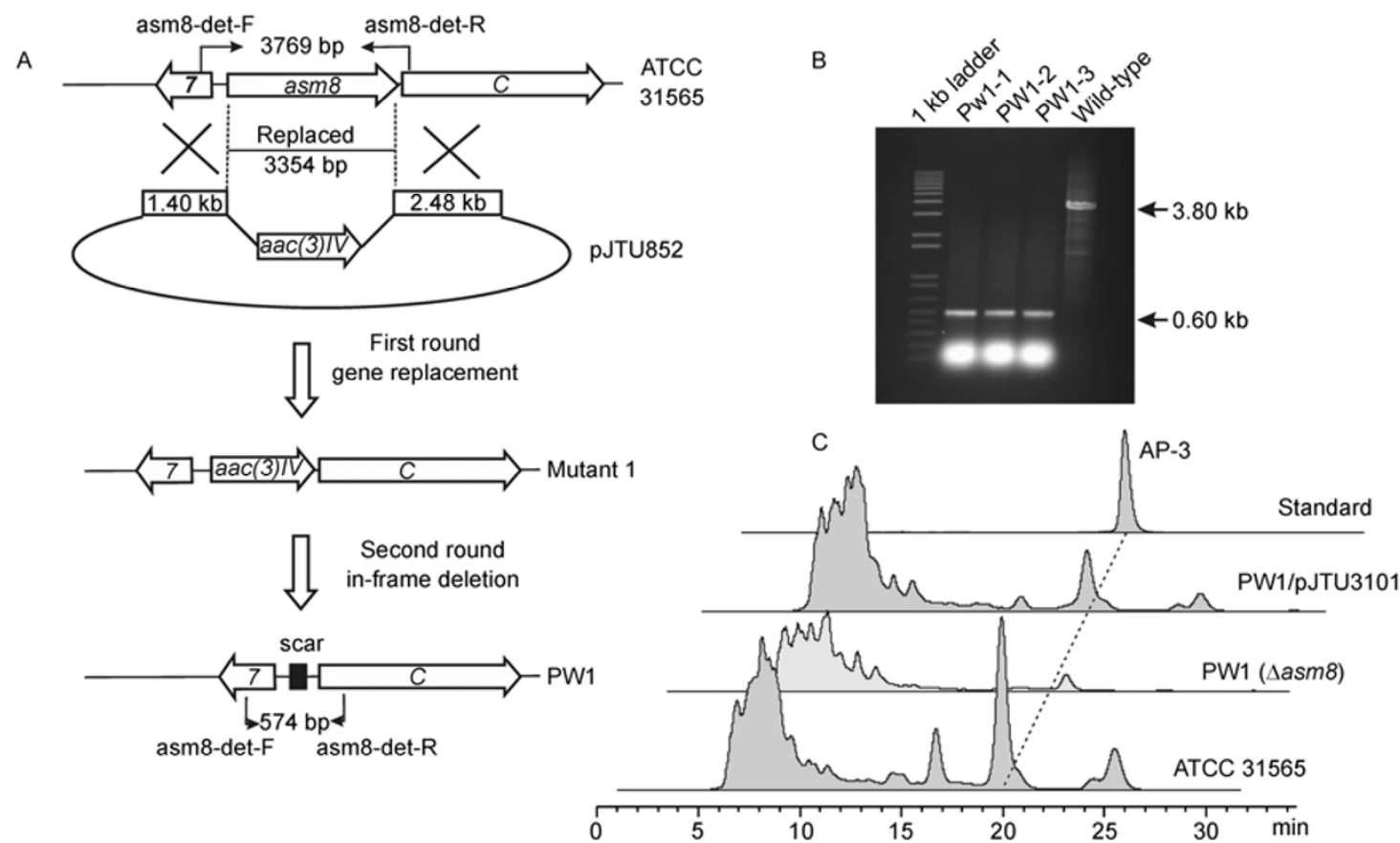

Figure 2 Schematic representation of asm8 inactivation and results of HPLC analysis of antibiotic production. A, Schematic representation of asm8 disruption. B, PCR analysis of wild-type and the PW1 mutant using primers asm8-Det-F and asm8-Det-R. C, HPLC analysis of the fermentation broths of wild-type ATCC 31565, mutant PW1, and PW1 complemented with cloned asm8 on pJTU3101. The peaks corresponding to AP-3 are marked by a dotted line.

with a cloned 3.80-kb DNA fragment containing asm8 and its 280-bp upstream region. Plasmid pJTU3101, carrying the $3.80-\mathrm{kb}$ fragment, was introduced into PW1 by conjugation. AP-3 productivity in the complemented mutant strain was restored to $30 \%$ of the wild-type (ATCC 31565) level (Figure 2C). The successful trans-complementation of PW1 verified that $a s m 8$ is a pathway-specific activator of ansamitocin biosynthesis, and that the upstream 280-bp region of asm8 contains the native promoter in ATCC 31565.

\subsection{Severely reduced transcription of AHBA biosyn- thetic genes in PW1}

The effect of asm8 mutation on ansamitocin production was further analyzed at the transcription level by qRT-PCR. The 45 genes putatively related to ansamitocin biosynthesis were chosen for transcription analysis. Total RNA from two-day cultures of the wild-type and PW1 mutant were extracted and analyzed by qRT-PCR. Surprisingly, the transcription levels of asm23, -24, -43, -44, -45, -46, and -47 were severely reduced in PW1, whereas transcription levels of the genes responsible for polyketide assembly, methoxymalonyl-ACP biosynthesis, post-PKS modification, and regulation were not affected. Other than asm46, the genes showing reduced expression in PW1 are all involved in AHBA biosynthesis [20] (Figure 3). In addition, disruption of asm46 had no effect on AP-3 production (data not shown).

\subsection{Feeding with AHBA restored ansamitocin biosyn- thesis}

The qRT-PCR analysis suggested that Asm8 specifically regulates the expression of the AHBA biosynthetic genes, rather than acting on the transcription of other biosynthetic genes. More supporting evidence was obtained from feeding experiments, whereby exogenous AHBA was fed to cultures of the PW1 mutant strain. PW1 was cultivated in fermentation medium supplemented with 1, 10, or $20 \mathrm{mg} \mathrm{L}^{-1}$ AHBA. As shown in Figure 4, the production level of AP-3 in PW1 gradually increased in proportion to the amount of exogenous AHBA added to the fermentation broths.

\subsection{Identification of asm8 homologs and two new an- samitocin producers}

BLAST analysis of Asm8 against the database identified homologous protein Amir_3178 in Actinosynnema mirum DSM 43827, which had an identity of $77 \%$. As the genome sequence of DSM 43827 is available [21], the flanking sequences of asm8 and amir_3178 were compared. A strikingly high level of sequence similarity and organization, especially for the ansamitocin biosynthetic genes, was observed between ATCC 31565 and DSM 43827 (Figure S1 in Supporting Information). Moreover, genes homologous to the AHBA-specific genes asm43-47 were also identified, not only in DSM 43827, but also in A. pretiosum ATCC 31280 [22], a strain known for ansamycin-type macbecin 


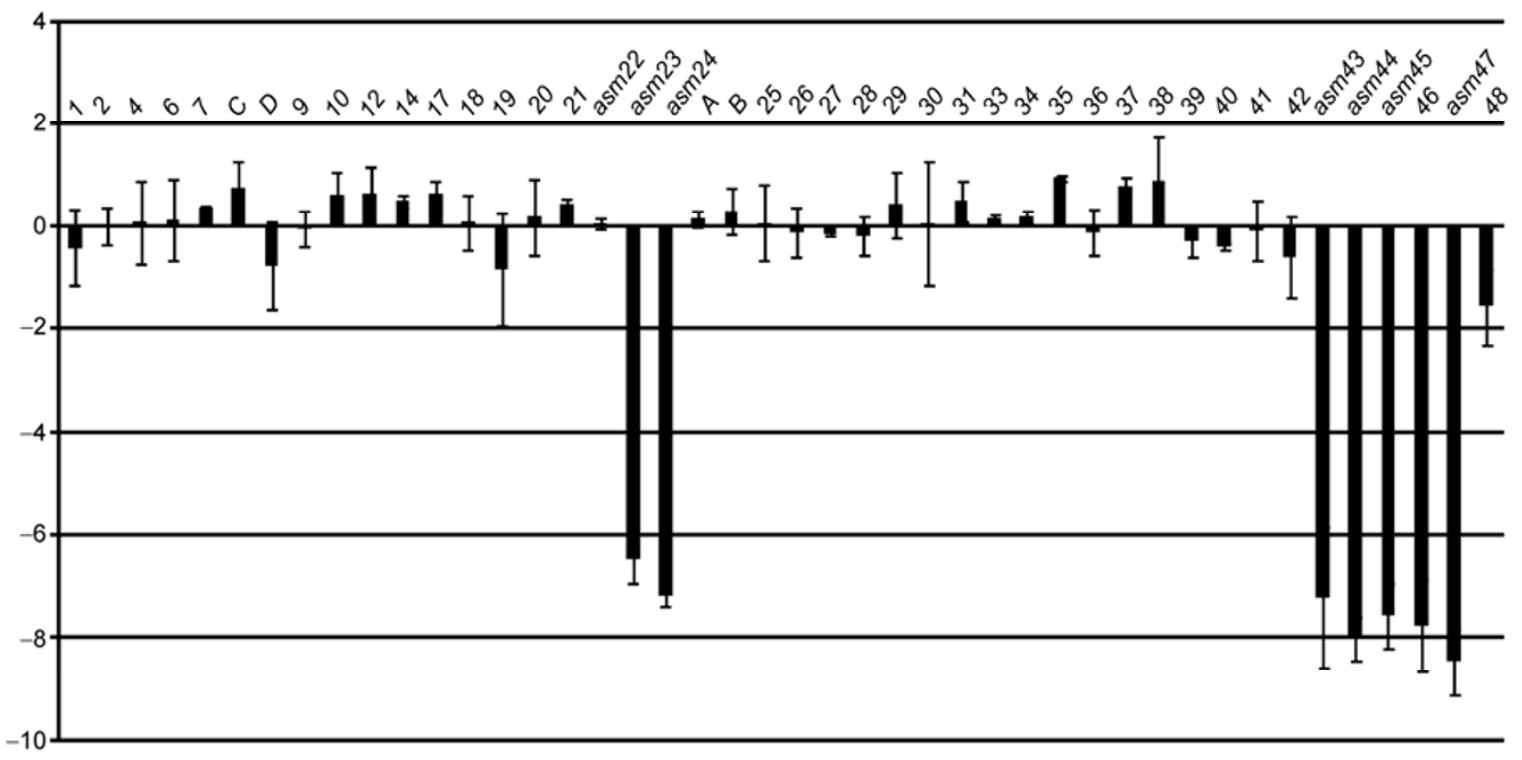

Figure 3 Transcriptional analysis of ansamitocin biosynthetic genes in PW1 compared with ATCC 31565. Transcription levels were determined by qRT-PCR, and putative AHBA biosynthesis genes are indicated in bold.

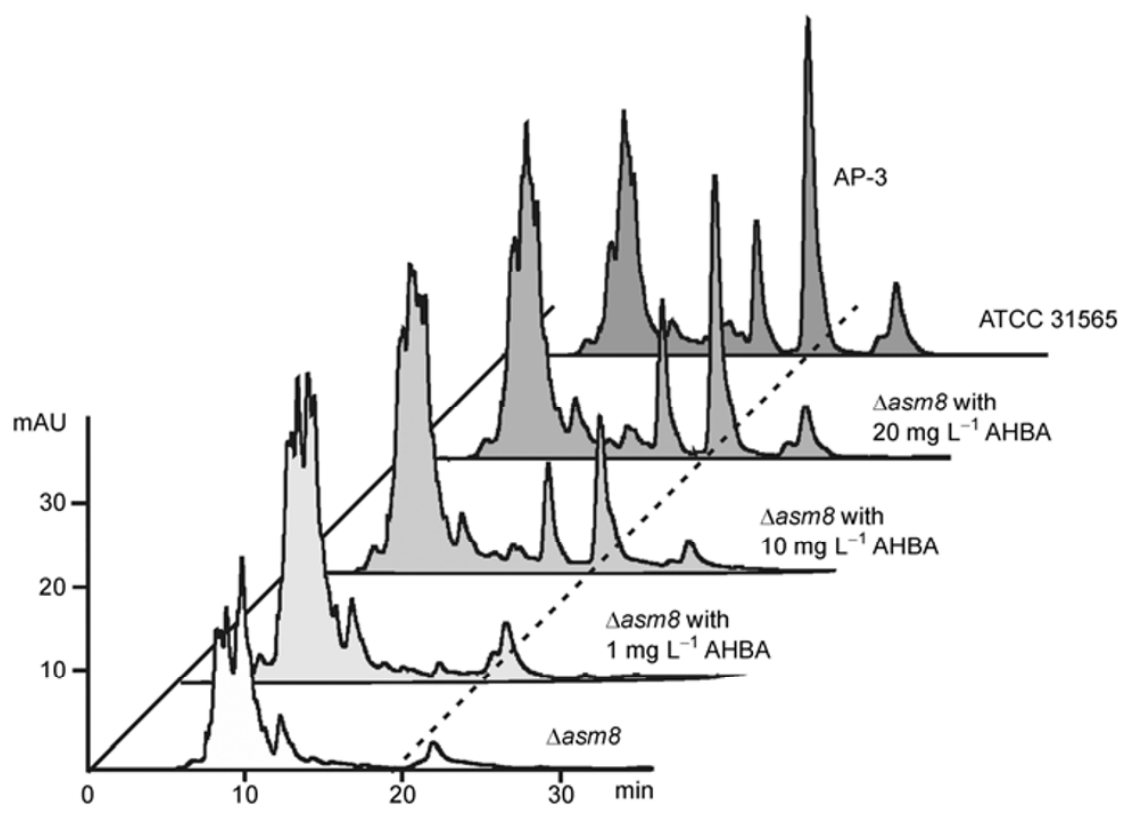

Figure 4 Feeding of exogenous AHBA to the PW1 mutant. The level of AP-3 production by the PW1 mutant was analyzed by HPLC when PW1 was cultivated in fermentation medium supplemented with $0,1,10$, or $20 \mathrm{mg} \mathrm{L}^{-1}$ exogenous AHBA.

production (Figure S1). This gene organization suggested that the ansamitocin biosynthetic gene cluster may be present in the ATCC 31280 genome.

Several pairs of primers specific for the ansamitocin biosynthetic genes were used to try and PCR amplify the corresponding genes from ATCC 31280 . The resultant products were sequenced and found to be identical to the ho- mologous genes in ATCC 31565 (data not shown). In addition, all the three Actinosynnema strains were cultivated on YMG agar plates for $7 \mathrm{~d}$, and the plates were then extracted with ethyl acetate for LC-MS analysis. Interestingly, peaks with the same retention time and mass as AP-3 were detected in both ATCC 31280 and DSM 43827 (Figure 5A), confirming the identification of two new ansamitocin- 

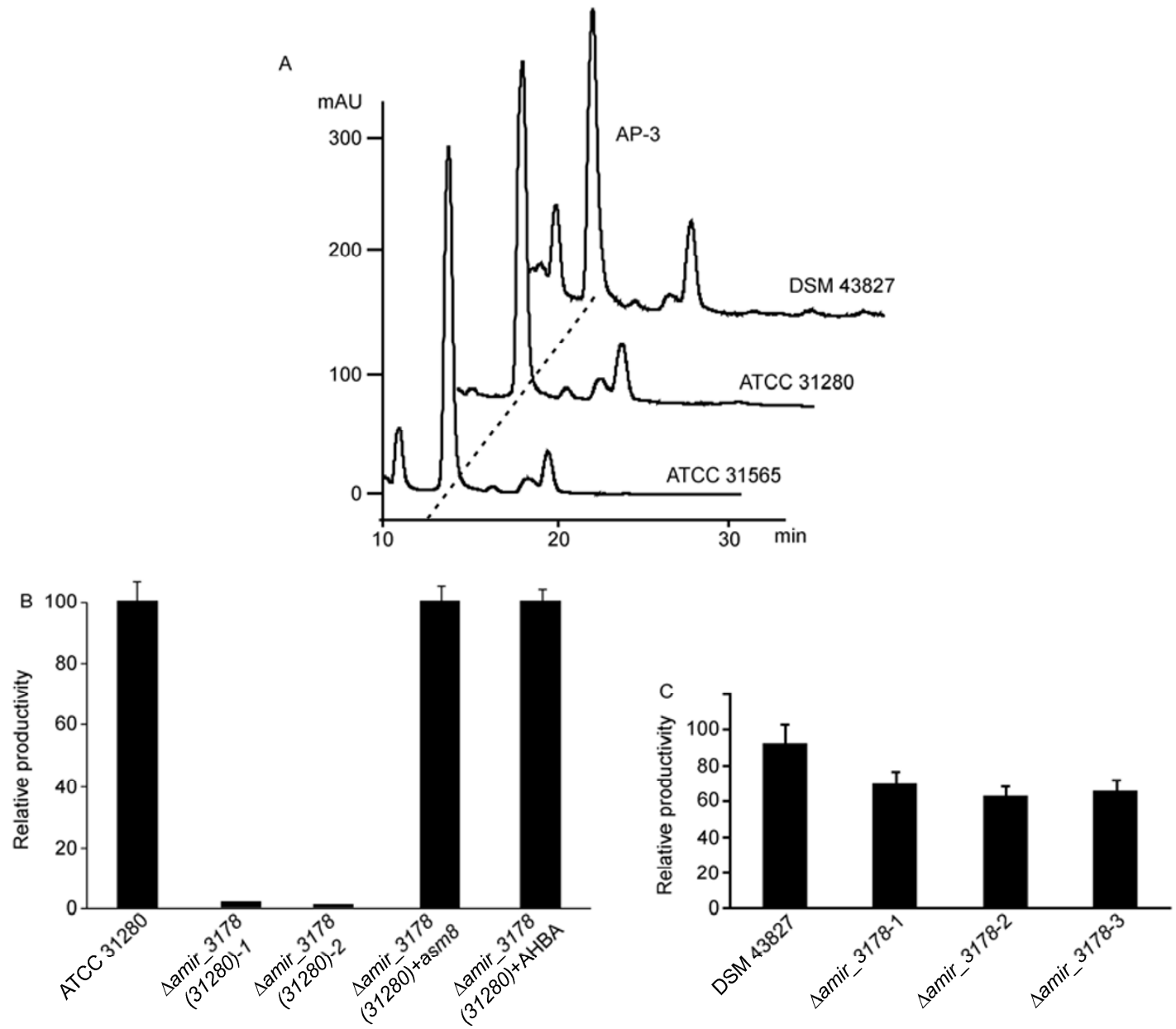

Figure 5 Detection of ansamitocins in the fermentation broths of ATCC 31280 and DSM 43827. A, LC-MS analysis of ansamitocins. The peaks corresponding to AP-3 are indicated by a dotted line. B, AP-3 production in ATCC 31280, an amir_3178(31280) mutant, an amir_3178(31280) mutant complemented with cloned asm8, and an amir_3178(31280) mutant supplemented with exogenous AHBA. Gene amir_3178(31280) was homologous to asm8 from ATCC 31280. C, AP-3 production in DSM 43827 and the amir_3178 mutants. Gene amir_3178 is homologous to asm8 from DSM 43827.

producing strains.

\subsection{Inactivation of genes homologous to asm8 in ATCC 31280 and DSM 43827}

In strain ATCC 31280, a gene homologous to asm8 was also identical to amir_3178 from DSM 43827, and was subsequently named amir_3178(31280). Gene amir_3178 (31280) was inactivated by gene replacement in ATCC31280 (Figure S2), and the production of AP-3 was completely abolished (Figure 5B). As expected, feeding with exogenous AHBA recovered the AP-3 productivity of the amir_3178(31280) mutant. A trans-complemented mutant with cloned asm8 expressed from plasmid pJTU3101 had restored AP-3 productivity.

Similar gene inactivation of asm8 homologous gene amir_3178 was also performed in DSM 43827 (Figure S3). Even though amir_3178 from DSM 43827 was identical to amir_3178(31280), the amir_3178 deletion mutant was surprisingly found to be capable of producing AP-3, albeit with a reduced yield of $60 \%-70 \%$ of the wild-type level (Figure 5C).

\section{Discussion}

The LAL family regulatory proteins are characterized by a LuxR-type pattern, consisting of a helix-turn-helix DNAbinding motif ( $\sim 65$ amino acids) at the $\mathrm{C}$ terminus, and a distinctive $\mathrm{P}$-loop at the $\mathrm{N}$-terminus, which is specific for an ATP-binding site. With a size of up to $116 \mathrm{kD}$, Asm8 fits the profile of LAL family proteins, and has a high degree of sequence similarity to a number of Streptomyces regulators, such as GdmRII from S. hygroscopicus 17997 [23], and AveR from S. avermitilis K139 [24], which are pathwayspecific transcriptional activators of geldanamycin biosynthesis and avermectin biosynthesis, respectively.

Our experiments demonstrated for the first time that the 
transcriptional activator gene asm8 is required for AP-3 production, and specifically controls the biosynthesis of the AHBA starter unit. Although most of the antibiotic biosynthetic gene clusters from actinomycetes have one or more regulatory genes [25], the presence of regulators controlling a complete set of genes with designated functions, such as precursor biosynthesis genes, is rare.

Lechner et al. [26] identified a LuxR regulatory gene, $s a l R 2$, in the salinosporamide biosynthetic gene cluster from Salinispora tropica; this gene is specifically involved in the formation of the halogenated precursor chloroethylmalonylCoA. When the salR2 mutant was supplemented with intermediate 4-chlorocrotonic acid, the productivity of salinosporamide was restored. In the clavulanic acid biosynthetic gene cluster, a LysR transcriptional regulator, ClaR, was specific for the expression of genes in the downstream steps of clavulanic acid production, i.e., in the conversion of clavaminic acid to clavulanic acid [27].

Transcriptional analysis using qRT-PCR determined that the transcription of seven genes was significantly reduced (Figure 3), six of which were implicated in AHBA biosynthesis (Figure 1A). asm46 was not related to ansamitocin biosynthesis (data not shown). Moreover, the transcription of asm22, encoding a putative kinase and homologous to rifN, was not affected. Even though homologs of asm 22 are present in all identified AHBA biosynthetic gene sets [28], its involvement in AHBA formation in ATCC 31565 needs to be further confirmed, either through gene inactivation or by in vitro enzymatic catalysis with the substrate kanosamine.

To date, the negative regulatory gene asm 2 and the positive regulatory gene asm 8 are the only two genes characterized among the nine putative regulatory genes within the previously sequenced region [6]. Recently, eight more regulatory genes were identified by sequencing the $30-\mathrm{kb}$ region between Cluster I and Cluster II (GenBank accession number of HQ441578) (Figure S1). The AP-3 yield was nearly doubled in a mutant with the $30-\mathrm{kb}$ region deleted (data not shown). Because there are 18 regulatory genes within the $120-\mathrm{kb}$ ansamitocin biosynthetic gene cluster, the biosynthesis of ansamitocin is assumed to be stringently regulated, which might cause difficulties for increasing the titer of ansamitocin.

Further investigation into the functions of other regulatory genes will assist with boosting the expression of ansamitocin biosynthetic genes, and ultimately increasing the titer. Already, introducing the cloned asm8 plasmid construct into the wild-type ATCC 31565 strain has been shown to increase the AP-3 titer. Moreover, the identification of ATCC 31280 and DSM 43827 as new ansamitocin producers may provide better starting candidates for genetic manipulation and titer improvement.

This work was supported by grants from the Ministry of Science and Technology of China (2012CB721005, 2012AA02A706, 2011ZX08009-001, 2012AA022107), the National Natural Science Foundation of China
(31070070, 31121064), Ministry of Education of China (20110073110048), and the SJTU-UM Collaborative Program.

1 Kupchan S M, Komoda Y, Court W A, et al. Maytansine, a novel antileukemic ansa macrolide from Maytenus ovatus. J Am Chem Soc, 1972, 94: 1354-1356

2 Higashide E, Asai M, Ootsu K, et al. Ansamitocin, a group of novel maytansinoid antibiotics with antitumour properties from Nocardia. Nature, 1977, 270: 721-722

3 Dhillon S. Everolimus in combination with exemestane: a review of its use in the treatment of patients with postmenopausal hormone receptor-positive, HER2-negative advanced breast cancer. Drugs, 2013, 73: 475-485

4 Kim C G, Yu T W, Fryhle C B, et al. 3-Amino-5-hydroxybenzoic acid synthase, the terminal enzyme in the formation of the precursor of $\mathrm{mC}_{7} \mathrm{~N}$ units in rifamycin and related antibiotics. J Biol Chem, 1998, 273: 6030-6040

5 Watanabe K, Rude M A, Walsh C T, et al. Engineered biosynthesis of an ansamycin polyketide precursor in Escherichia coli. Proc Natl Acad Sci USA, 2003, 100: 9774-9778

$6 \mathrm{Yu} \mathrm{T} \mathrm{W,} \mathrm{Bai} \mathrm{L} \mathrm{Q,} \mathrm{Clade} \mathrm{D,} \mathrm{et} \mathrm{al.} \mathrm{The} \mathrm{biosynthetic} \mathrm{gene} \mathrm{cluster} \mathrm{of} \mathrm{the}$ maytansinoid antitumor agent ansamitocin from Actinosynnema pretiosum. Proc Natl Acad Sci USA, 2002, 99: 7968-7973

7 Wu Y, Kang Q, Shang G, et al. $N$-methylation of the amide bond by methyltransferase asm10 in ansamitocin biosynthesis. Chembiochem, 2011, 12: 1759-1766

8 Li Y, Zhao P, Kang Q, et al. Dual carbamoylations on the polyketide and glycosyl moiety by asm 21 result in extended ansamitocin biosynthesis. Chem Biol, 2011, 18: 1571-1580

9 Spiteller P, Bai L, Shang G, et al. The post-polyketide synthase modification steps in the biosynthesis of the antitumor agent ansamitocin by Actinosynnema pretiosum. J Am Chem Soc, 2003, 125: 14236-14237

10 Bandi S, Kim Y, Chang Y K, et al. Construction of asm2 deletion mutant of Actinosynnema pretiosum and medium optimization for ansamitocin P-3 production using statistical approach. J Microbiol Biotechn, 2006, 16: 1338-1346

$11 \mathrm{Ng} \mathrm{D}$, Chin H K, Wong V V T. Constitutive overexpression of asm2 and asm39 increases AP-3 production in the actinomycete Actinosynnema pretiosum. J Ind Microbiol Biot, 2009, 36: 1345-1351

12 Paget M S, Chamberlin L, Atrih A, et al. Evidence that the extracytoplasmic function sigma factor sigmaE is required for normal cell wall structure in Streptomyces coelicolor A3(2). J Bacteriol, 1999, 181: 204-211

13 He Y, Wang Z, Bai L, et al. Two pHZ1358-derivative vectors for efficient gene knockout in streptomyces. J Microbiol Biotechnol, 2010, 20: 678-682

14 Wilkinson C J, Hughes-Thomas Z A, Martin C J, et al. Increasing the efficiency of heterologous promoters in actinomycetes. J Mol Microbiol Biotechnol, 2002, 4: 417-4126

15 Flett F, Mersinias V, Smith C P. High efficiency intergeneric conjugal transfer of plasmid DNA from Escherichia coli to methyl DNA-restricting streptomycetes. FEMS Microbiol Lett, 1997, 155: 223-229

16 Sambrook J, Fritsch E F, Maniatis T. Molecular Cloning: a Laboratory Manual, 2nd ed. New York: Cold Spring Harbor, 1989

17 Kieser T, Bibb M J, Buttner M J, et al. Practical Streptomyces Genetics. Norwich: The John Innes Foundation, 2000

18 Gust B, Challis G L, Fowler K, et al. PCR-targeted Streptomyces gene replacement identifies a protein domain needed for biosynthesis of the sesquiterpene soil odor geosmin. Proc Natl Acad Sci USA, 2003, 100: 1541-1546

19 Zhao P, Bai L, Ma J, et al. Amide $N$-glycosylation by Asm25, an $N$-glycosyltransferase of ansamitocins. Chem Biol, 2008, 15: 863874

20 Cassady J M, Chan K K, Floss H G, et al. Recent developments in the maytansinoid antitumor agents. Chem Pharm Bull, 2004, 52: 1-26

21 Land M L A, Mayilraj S, Chen F, et al. Complete genome sequence 
of Actinosynnema mirum type strain (101T). Stand Genom Sci, 2009, 1: 46-53

22 Ono Y, Kozai Y, Ootsu K. Antitumor and cytocidal activities of a newly isolated benzenoid ansamycin, macbecin I. Gann, 1982, 73: 938-944

23 He W Q, Lei J, Liu Y Y, et al. The LuxR family members GdmRI and GdmRII are positive regulators of geldanamycin biosynthesis in Streptomyces hygroscopicus 17997. Arch Microbiol, 2008, 189: 501-510

24 Kitani S, Ikeda H, Sakamoto T, et al. Characterization of a regulatory gene, aveR, for the biosynthesis of avermectin in Streptomyces avermitilis. Appl Microbiol Biot, 2009, 82: 1089-1096
25 Liu G, Chater K F, Chandra G, et al. Molecular regulation of antibiotic biosynthesis in streptomyces. Microbiol Mol Biol Rev, 2013, 77: 112-143

26 Lechner A, Eustaquio A S, Gulder T A, et al. Selective overproduction of the proteasome inhibitor salinosporamide A via precursor pathway regulation. Chem Biol, 2011, 18: 1527-1536

27 Paradkar A S, Aidoo K A, Jensen S E. A pathway-specific transcriptional activator regulates late steps of clavulanic acid biosynthesis in Streptomyces clavuligerus. Mol Microbiol, 1998, 27: 831-843

28 Kang Q, Shen Y, Bai L. Biosynthesis of 3,5-AHBA-derived natural products. Nat Prod Rep, 2012, 29: 243-263

Open Access This article is distributed under the terms of the Creative Commons Attribution License which permits any use, distribution, and reproduction in any medium, provided the original author(s) and source are credited.

\section{Supporting Information}

Table S1 Strains and plasmids used in this study

Figure S1 Gene organization in the regions flanking asm 8 and its homologs in A. pretiosum ATCC 31565 and ATCC 31280 , and in A. mirum DSM 43827.

Figure S2 Disruption of amir_3178(31280) in ATCC 31280. A, Schematic representation of the inactivation of amir_3178(31280) in ATCC 31280. amir_3178(31280) was first replaced by an apramycin resistance gene, aac(3)IV, through Redirect Technology, and then replaced by an 81-bp scar fragment. B, PCR amplification of the DNA templates from amir_3178 (31280) mutants, designated PW11.

Figure S3 Deletion of amir_3178 in DSM 43827. A, Schematic representation of the inactivation of amir_3178 in DSM 43827 . Gene amir_3178 was replaced by an apramycin resistance gene, aac(3)IV, using Redirect Technology. B, PCR amplification with two pairs of primers (3178-det-F1/R1 and 3178-det-F2/R2) using genomic DNA from the amir_3178 mutant, PW21, as the template.

The supporting information is available online at life.scichina.com and www.springerlink.com. The supporting materials are published as submitted, without typesetting or editing. The responsibility for scientific accuracy and content remains entirely with the authors. 